\title{
ON THE MEAN ERGODIC THEOREM FOR SUBSEQUENCES
}

BY J. R. BLUM AND D. L. HANSON

Communicated by J. C. Oxtoby, April 11, 1960

1. Let $\Omega$ be a set, $Q$ a $\sigma$-algebra of subsets of $\Omega$, and $P$ a probability measure defined on $Q$, i.e. $P$ is a nonnegative completely additive set function defined on $a$, with $P(\Omega)=1$. If $p$ is a number with $1 \leqq p<\infty$ we shall denote as is usual the Banach space of measurable functions $f$ for which

$$
\int_{\Omega}|f|^{p} d P<\infty \quad \text { by } L_{p}(\Omega, \propto, P) \text {. }
$$

Let $T$ be a transformation mapping $\Omega$ onto $\Omega$ which is $1-1$ and bimeasurable, i.e. if $A \in \mathbb{Q}$ then

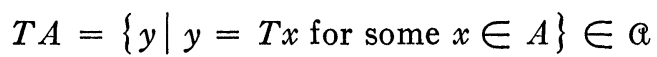

and

$$
T^{-1} A=\{y \mid T y \in A\} \in \mathfrak{Q} .
$$

With these assumptions we have $T^{n}$ defined for every integer $n$ as a 1-1, onto, bimeasurable transformation. Henceforth we shall assume that every set considered is measurable, i.e. an element of $a$. We shall say that $P$ is invariant if $P(A)=P(T A)$ for every set $A, P$ is ergodic if $P$ is invariant and if $P\left(\bigcup_{n=-\infty}^{\infty} T^{n} A\right)=1$ for every set $A$ for which $P(A)>0$, and finally $P$ is strongly mixing if $P$ is invariant and

$$
\lim _{n} P\left[\left(T^{n} A\right) \cap B\right]=P(A) P(B)
$$

for every pair of sets $A, B$. It is a consequence of the individual ergodic theorem that if $P$ is invariant then $P$ is ergodic if and only if

$$
\lim _{n} \frac{1}{n} \sum_{i=0}^{n-1} \mathrm{P}\left[\left(T^{i} A\right) \cap B\right]=P(A) P(B)
$$

for every pair of sets $A, B$. Thus every strongly mixing measure is ergodic.

It is the object of this paper to show that the strong mixing condition is equivalent to the validity of the mean ergodic theorem for every $L_{p}$ space and every subsequence $\left\{T^{k_{n}}\right\}$ of the sequence $\left\{T^{n}\right\}$. More precisely let $p$ be a number with $1 \leqq p<\infty$, let $f \in L_{p}(\Omega, a, P)$ 
and let $\left\{k_{n}\right\}$ be a sequence of distinct integers with $\lim _{n} k_{n}=\infty$. Then we shall show that if $P$ is strongly mixing we have

$$
\lim _{n} \frac{1}{n} \sum_{i=1}^{n} f\left(T^{k_{i}} x\right)=\int_{\Omega} f d P
$$

in $L_{p}$ norm. Conversely it is also true that this conclusion implies that $P$ is strongly mixing.

The main interest of this result lies in the fact that in previous ergodic theorems, both of the individual and the mean type, it is assumed that the sequence of operators over which one averages forms a semigroup. This property can be dispensed with if one is willing to assume the strong mixing property.

2. We shall need several lemmas before proving the main result.

Lemma 1. Let $\left\{C_{i, j} ; i, j \geqq 1\right\}$ be a bounded sequence of numbers such that $\lim _{|i-j| \rightarrow \infty} C_{i, j}=0$. Then

$$
\lim _{n} \frac{1}{n^{2}} \sum_{i, j=1}^{n} C_{i, j}=0
$$

Proof. Let $C=\sup _{i, j}\left|C_{i, j}\right|$ and let $\epsilon$ be a positive number. Choose $M$ so that $\left|C_{i, j}\right| \leqq \epsilon$ for $|i-j|>M$. Then for $n>M$ we have

$$
\left|\frac{1}{n^{2}} \sum_{i, j=1}^{n} C_{i j}\right| \leqq \frac{(2 M+1) C}{n}+\frac{1}{n^{2}} \sum_{|i-j|>M}\left|C_{i, j}\right|
$$

and the lemma follows.

Lemma 2. Let $P$ be strongly mixing and let $f_{A}(x)$ be the set characteristic function of $a$ set $A$. Then

$$
\lim _{n} \int_{\Omega}\left|\frac{1}{n} \sum_{i=1}^{n} f_{A}\left(T^{k^{i} x}\right)-P(A)\right|^{2} d P=0
$$

for every strictly increasing sequence $\left\{k_{n}\right\}$ of integers.

Proof. Let $\left\{k_{n}\right\}$ be such a sequence. Then

$$
\begin{aligned}
\int_{\Omega}\left|\frac{1}{n} \sum_{i=1}^{n} f_{A}\left(T^{k_{i}} x\right)-P(A)\right|^{2} d P \\
\quad=\frac{1}{n^{2}} \sum_{i, j=1}^{n} \int_{\Omega}\left[f_{A}\left(T^{k_{i}} x\right)-P(A)\right]\left[f_{A}\left(T^{k_{i}} x\right)-P(A)\right] d P \\
=\frac{1}{n^{2}} \sum_{i, j=1}^{n}\left\{P\left[\left(T^{k_{i}} A\right) \cap\left(T^{k_{i}} A\right)\right]-P^{2}(A)\right\}
\end{aligned}
$$


since $P$ is invariant. The conclusion now follows from the hypothesis and Lemma 1 by letting $C_{i, j}=P\left[\left(T^{k_{i}} A\right) \cap\left(T^{k_{j}} A\right)\right]-P^{2}(A)$. Note that

$$
P\left[\left(T^{k_{i}} A\right) \cap\left(T^{k_{j}} A\right)\right]=P\left[\left(T^{\left|k_{i}-k_{j}\right|} A\right) \cap A\right] .
$$

Lemma 3. Lemma 2 remains valid if we replace $f_{A}(x)$ by a simple function and $P(A)$ by $\int_{\Omega} f(x) d P$.

Proof. The proof is accomplished by simple algebraic manipulations and the application of Lemma 2.

LeMma 4. Lemma 3 remains valid if we replace $L_{2}$ convergence by $L_{p}$ convergence where $p$ is any number with $1 \leqq p<\infty$.

Proof. For $p=1$ the lemma follows from Lemma 3 and the Hölder inequality. Let $p>1$ and let $g(x)$ be a bounded measurable function with bound $M$. Then

$$
\begin{aligned}
\int_{\Omega}|g(x)|^{p} d P & \leqq \int_{\{|g| \leq 1\}}|g| d P+M^{p} \int_{\{|| \mid>1\}} d P \\
& \leqq\left(1+M^{p}\right) \int_{\Omega}|g| d P .
\end{aligned}
$$

The lemma now follows from the fact that it holds for $p=1$, and if $f$ is a simple function then

$$
\frac{1}{n} \sum_{i=1}^{n} f\left(T^{k} x\right)-\int_{\Omega} f d P
$$

is bounded uniformly in $n$.

THEOREM. $P$ is strongly mixing if and only if for every number $p$ with $1 \leqq p<\infty$, every strictly increasing sequence of integers, and every $f \in L_{p}(\Omega, Q, P)$ we have

$$
\lim _{n} \int_{\Omega}\left|\frac{1}{n} \sum_{i=1}^{n} f\left(T^{k i} x\right)-\int_{\Omega} f d P\right|^{p} d P=0 .
$$

Proof. Assume that $P$ is strongly mixing. Let $f \in L_{p}(\Omega, Q, P)$ for some $p$, and let $\left\{k_{n}\right\}$ be a strictly increasing sequence of integers. It is clearly sufficient to prove the conclusion when $f$ is nonnegative and we shall assume this is the case. Then there exists a nondecreasing sequence $\left\{f_{m}\right\}$ of simple functions such that $\lim _{m}\left|f_{m}-f\right|=0$ almost everywhere. Let $\|g\|_{p}$ be the $L_{p}$ norm of the function $g$. Then 


$$
\begin{array}{r}
\left\|\frac{1}{n} \sum_{i=1}^{n} f\left(T^{k i} x\right)-\int_{\Omega} f d P\right\|_{p} \leqq\left\|\frac{1}{n} \sum_{i=1}^{n} f\left(T^{k i} x\right)-\frac{1}{n} \sum_{i=1}^{n} f_{m}\left(T^{k i} x\right)\right\|_{p} \\
+\left\|\frac{1}{n} \sum_{i=1}^{n} f_{m}\left(T^{k^{i}} x\right)-\int_{\Omega} f_{m} d P\right\|_{p}+\left\|\int_{\Omega} f_{m} d P-\int_{\Omega} f d P\right\|_{p}
\end{array}
$$

and each of the last three terms can be made small by choosing $m$ and $n$ sufficiently large, from Lemma 4 and the monotone convergence theorem.

Conversely suppose the second condition of the theorem holds. Let $A$ and $B$ be sets with set characteristic functions $f_{A}$ and $f_{B}$ and suppose $P\left[\left(T^{-k_{n}} A\right) \cap B\right]-P(A) P(B) \geqq \epsilon>0$ for a sequence of integers $\left\{k_{n}\right\}$. (A similar argument applies if $P\left[\left(T^{-k_{n}} A\right) \cap B\right]-P(A) P(B)$ $\leqq-\epsilon<0$.) Then

$$
\frac{1}{n} \sum_{i=1}^{n} P\left[\left(T^{-k_{i}} A\right) \cap B\right]-P(A) P(B) \geqq \epsilon
$$

for all $n$. But

$$
\begin{aligned}
& \frac{1}{n} \sum_{i=1}^{n} P\left[\left(T^{-k_{i}} A\right) \cap B\right]-P(A) P(B) \\
& =\int_{\Omega} f_{B}(x)\left[\frac{1}{n} \sum_{i=1}^{n} f_{A}\left(T^{k_{i}} x\right)-P(A)\right] d P \leqq\left\|\frac{1}{n} \sum_{i=1}^{n} f_{A}\left(T^{k_{i}} x\right)-P(A)\right\|_{1}
\end{aligned}
$$

which approaches zero. The theorem is proved.

It is likely that the theorem may be generalized in the same direction that the mean ergodic theorem has been generalized, i.e. by considering more general operators. More interestingly, while the authors have partial results for the individual ergodic theorem, the question of whether the individual ergodic theorem holds for all subsequences in the case of strong mixing measures remains open.

Sandia Corporation and

International Business Machines Corporation 